\title{
Po-Shen Loh Interview
}

Editor's Note: After this interview, Loh's US team placed first in the July 2016 International Math Olympiad in Hong Kong, including two perfect scores, followed by South Korea and China. The Leader (coach) of the Korean team, Yongjin Song, praised to us Loh's extraordinary talent and kindness as Leader of the US team, in the face of what he had considered an unbeatable Chinese team.

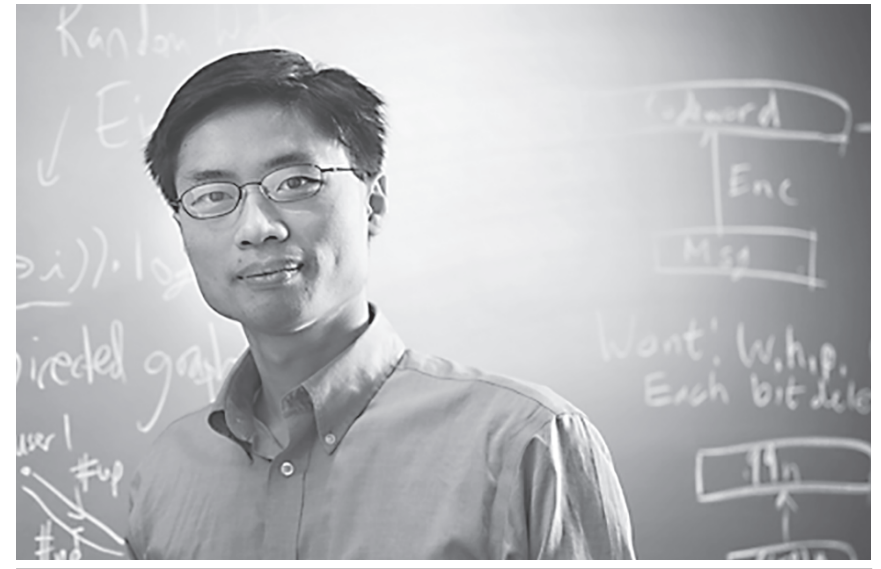

Po-Shen Loh is associate professor of mathematics at Carnegie Mellon University, national lead coach of the USA International Mathematical Olympiad (IMO) team, and founder of expi i . com. His e-mail address is ploh@cmu .edu.

Diaz-Lopez: When did you know you wanted to be a mathematician?

Loh: I have always liked mathematics, and I enjoy doing as much as I can with mathematics. I was interested in competition mathematics before college, but it was in the very first year of college, when I took an abstract algebra class from an excellent professor, when I suddenly found that this abstract mathematics is really beautiful, really fun; I loved the structure and that's when I got hooked.

Diaz-Lopez: Who encouraged you?

Loh: At an early age I was just not very good at anything else, so by default I was a mathematician, but at a later age certainly my PhD advisor Benjamin Sudakov encouraged me. He is a very interesting person who helped migrate my way of mathematical thinking from a very amateur stage to what it is now.

DOI: http://dx.doi.org/10.1090/noti1421
Diaz-Lopez: How would you describe your research to a graduate student?

Loh: I like to think about interesting questions. These questions are usually in the area of discrete mathematics because that happens to be where I have the most training. I prefer questions that come from extremal combinatorics, which is an area very heavily influenced by the work of Paul Erdős. It often combines different areas of mathematics. For example, it brings together probability and combinatorics. But ultimately what attracts me the most to a question is if the question seems inviting in some way and if it seems like there might be more you can learn by working on it.

Diaz-Lopez: What theorem are you most proud of?

Loh: It is very hard for me to identify one theorem because every theorem is a victory after a long fight, so instead I will mention the latest thing I am working on. Suppose I have two ordered triples of whole numbers; we say that one triple is bigger than the other if it is bigger in at least two coordinates. For example, $(1,3,6)$ is bigger than $(3,2,5)$ because it is bigger in the second and third coordinates. Now suppose I want to write down a long sequence of triples such that all the entries are integers from one to some parameter $n$, and I want to have the property that if I compare any triple to any other triple that comes later, the later one is bigger (in at least two coordinates). The question is simple: What is the maximum possible length of such sequence?

Simple problem, right? That's what I thought. I came across this question when I was working in an area of mathematics called Ramsey theory, and I was doing something else, something quite natural in Ramsey theory, and it boils down to this problem that I just described. When I got to that stage I was very happy. I thought I would be done in an hour. I thought it had to be trivial, it's so easy to describe, surely it will fall from some simple argument like the pigeonhole principle, and I will be done. I wasn't done in one hour, actually I'm still not done, and in fact there have been quite a few people who tried it and they also are not done. I was able to prove that the length of 
such sequence is at most $n^{2} / \log ^{*}(n)$. $\log ^{*}(n)$ is a strange function; it's the inverse function of the power function. The power function of $k$ is $2^{2^{2.2^{2}}}(k$ times), which grows enormously. The inverse function still grows but extremely slowly.

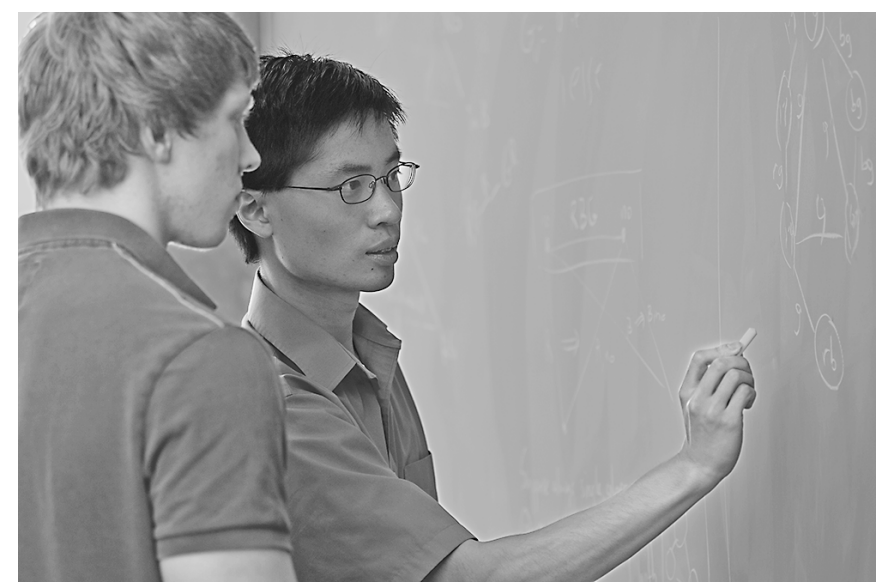

Po-Shen Loh works with a student.

This question is very interesting because it relates to a bunch of different types of questions in combinatorics. It reaches into an area influenced by Szemerédi and uses a result of Ruzsa and Szemerédi. This strange function $\log ^{*}(n)$ comes from that type of mathematics. I particularly like this result because it poses another twist in that general area where you have a beautiful interplay between combinatorics, probability, geometry, and number theory.

Diaz-Lopez: What advice do you have for graduate students?

Loh: Choosing the right advisor is extremely important. I was fortunate: I really enjoyed working with Benny [Sudakov]. I saw him quite frequently. I met with him more than once a day, although that is a bit unusual. This is because I liked doing mathematics in the common area, so he would just keep coming back around for coffee or something, and he would ask what's going on, what's the new result, so-Oh boy!, there better be a new result. At the same time he is a very good-hearted person. I would say the advisor is the most important thing.

Diaz-Lopez: All mathematicians feel discouraged occasionally. How do you deal with discouragement?

Loh: It's more of an issue if you feel pressure to produce something quickly. In general, whenever you have such pressure then you have that sense of disappointment. I generally try not to force myself to be in that situation. It's better to be consistently producing to avoid being in that situation. Additionally, I said I like to think about interesting questions, so if I solve a problem too fast then it was not that interesting in the first place. On the other hand, if it is interesting I don't mind sitting in a comfortable chair and playing with it. It's like staring at a diamond. You keep looking at it from different angles, it's beautiful, you admire it. In that sense I don't feel frustrated to not solve a problem, because it was supposed to be hard anyway.

Diaz-Lopez: You have won several honors and awards. Which one has been the most meaningful and why?

Loh: I don't dwell on honors. I've always gone to the next big challenge. So for me it's always about the hunt. If you got the trophy it's just going to sit on the wall. Certain things influenced my life but did not affect my psychology. For example, when I was in high school, I happened to be on the International Math Olympiad team for the USA. Among high school students it is very hard to distinguish talent, so whether we like it or not, colleges use this sort of information, and that certainly opened doors in my life. But I don't think that's what led me to think I'm good at math. If anything it taught me that there are many people out there better at math than I am.

\section{Expii}

Diaz-Lopez: One of the projects you are working on is Expii.

Loh: The history of Expii is very interesting. I became the coach of the USA Math Olympiad team about two years ago. I was thinking about what my strategy would be and considering alternatives. One easy thing to do would have been to identify the top 100 people in the USA and train them really hard. That's not my style. I wanted to do something that would help more people. So another strategy was to boost the baseline of mathematical capabilities in the USA and around the world. Expii is an attempt to give everybody automated tutoring in your pocket. Our goal is to do massive global education, to educate all 7 billion people on Earth. The idea behind it is to use smartphones to deliver automatic methods on topics in mathematics and science. There are actually ten thousand topics, and on each topic we are crowdsourcing the script of how you would tutor that topic. Actually, it is stronger than what I just described: it is not only crowdsourcing one way of teaching every topic, it's crowdsourcing the multiple ways, voices, and styles of explaining every topic, with voting helping discern which ones are the most popular. This is like Math Overflow. Essentially we are using the voting to identify what are the most engaging ways to explain all the topics. And being engaging is important, because if your goal is to get more people interested in mathematical sciences, then you want to have explanations that are fun and interesting. We are using the voting to do global statistical testing to find out what are the most exciting ways to explain other things. That's what we have already built.

There are more features we are continuing to build. For example, we recently released Expii Solve, which I hope will be useful to the general public. Expii Solve is an area of Expii where every week I am releasing a set of five interesting math problems for people to think about. These are five questions on an exponential difficulty scale. The first question is designed so that the average person can understand it. This part is to try to get more people interested in mathematics. For example, this is a question we posted:

You are walking through Madison, Wisconsin, and you see gallons of milk lined up all the way across the city-a 


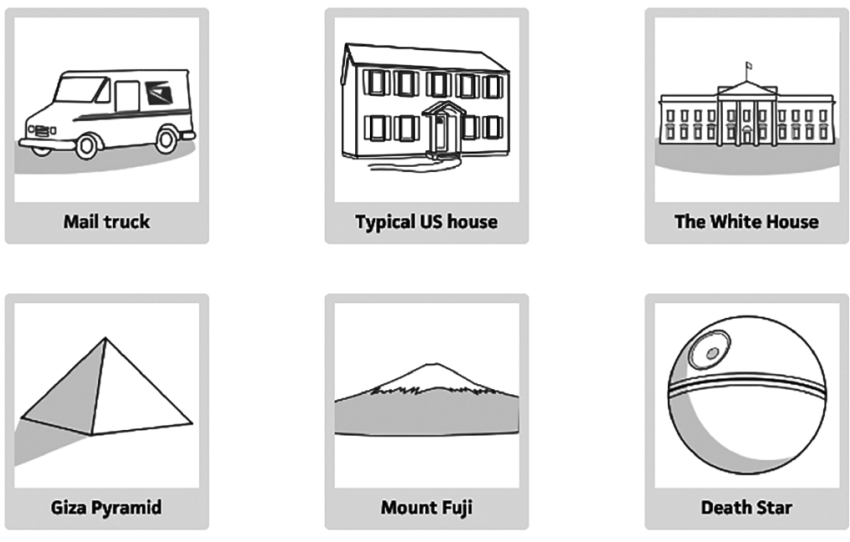

How big a container would be needed to hold gallons of milk lined up for twenty miles?

total of 20 miles long. If all the milk were poured into one big container, how big would the container need to be?

This is a question that most people can grasp, and it is designed in a way to get people interested in math in the sense that it is very visual and understandable. For this question, most people will guess the answer is the pyramid, which is too big. The second most popular answer is the White House, which is also too big. The correct answer is a typical US house, which is actually surprising. This is our vision, that everyone can approach question one, but it's designed so that even people who know math could get it wrong. The question is supposed to challenge the intuition and hopefully hook people into the math.

\section{"I will rate the success of the Olympiad program by whether the participants end up being successful in life."}

Of course then questions 2, 3, 4, and 5 become successively more difficult. The reason that we built this is to motivate people and give them resources to try and push a little further in math.

\section{International Math Olympiad}

Diaz-Lopez: Let me switch to another project. You have been involved in the USA Math Olympiad program since 2002. Last year you had a major achievement when the USA team won the International Math Olympiad. Why are you involved in this project and what else would you like to achieve?

Loh: First of all, I should say that I don't feel like I achieved anything in 2015. I didn't score any of the points. The team achieved this, and they get all the credit. Back to the question, I like the project because I've always liked education, and I like helping people achieve as much as they can. I happened to go through that pipeline myself as a student, and I benefited a lot from it. I was exposed to a lot of interesting mathematics and met many interesting people, so in some sense being the national head coach is a way of giving back. I've always felt the responsibility to give back to the various places that helped me at some point and the Math Olympiad circuit is a huge one.

I will rate the success of the Olympiad program by whether the participants end up being successful people. By this I am not talking about winning the Olympiad; I am talking about being successful in life. In fact, when I was offered to be the head coach, I warned the MAA [Mathematical Association of America] that we would most likely do worse because I would tell all the students that we were not going to focus on the Olympiad. We were going to think about the fact that there is a long way forward, there is lots of interesting mathematics, and at the national training program we would talk about higher mathematics as well as Olympiad mathematics and we were going to think very long term. The MAA was willing to take the risk on me anyway. We happened to win because of the fact that our students are really strong, but ultimately my goal is to have successful people come out.

Diaz-Lopez: Traditionally the students who participate in the Math Olympiad are males. Do you have any ideas on how to increase diversity?

Loh: Yes, I care a lot about this topic as well. There are some things being done right now. There are competitions where we encourage certain groups to participate. Those are generally started by people who have a designed goal, and they will try to boost the performance of students in these groups. I think that's great; the more of these things there are, the better. Within the Math Olympiad we have programs to bring girls into the National Math Olympiad training program to increase the number of girls who have exposure to this kind of proof-type mathematics. Now to

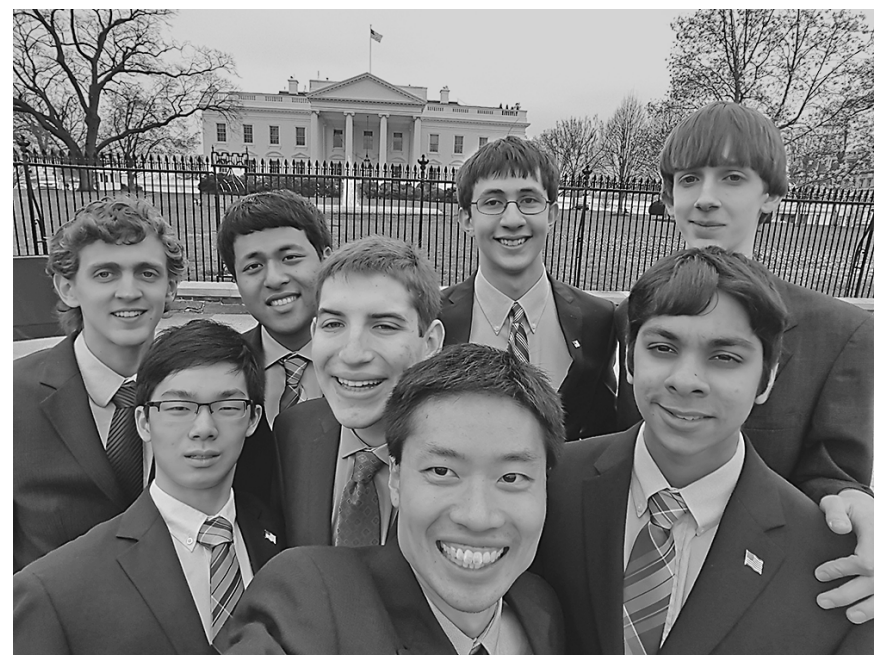

Po-Shen Loh taking a selfie with the 2015 USA team for the International Mathematical Olympiad, in front of the White House. That team placed first in the competition that year-the first time in twenty-one years that the USA team won the IMO. 
qualify for the International Math Olympiad, the first few exams are all purely computational, and then suddenly there is a proof-based exam. Most of the evaluation is done on questions that are different from Math Olympiad and future math questions. So we are making efforts toward changing that.

But personally what I think will be effective is Expii, and that's why I started building it. Integrated into the core of Expii is that for any given topic there are as many different explanations as anyone wants. My hope is that there will be more and more explanations that are good explanations and are written by people of diverse backgrounds. Peers learn well from peers. In fact, it may be that the best explanation of the quadratic formula is written by a woman. Wouldn't that be great? That would send a strong message. So I am hoping Expii will be effective at increasing diversity in the USA Math Olympiad program.

Diaz-Lopez: Any final comment?

Loh: I think math is extremely powerful. Everyone who has been trained in mathematics has been taught to think very creatively, to have a powerful way of reasoning abstractly that can build from lemma to lemma to lemma. Mathematics is the core of all this thinking and logic, so we should encourage everyone to study mathematics. In fact, I often make a joke that we should have a rebranding campaign, we should not call what we do "mathematics." Because right now if I go to the street and ask people "what is mathematics?" they will most likely say something like $7 * 6=42$. But mathematics is not memorizing algorithms, mathematics is thinking. So we should rebrand mathematics and call it thinking. Then nobody will be able to say, "I don't like thinking, I have never tried thinking."

\section{Credits}

Page 2 and 3 photos, courtesy of Carnegie Mellon University.

Page 4 images, courtesy of expi i.com.

Page 4 photo of Po-Shen Loh and the 2015 USA IMO team, courtesy of Po-Shen Loh.

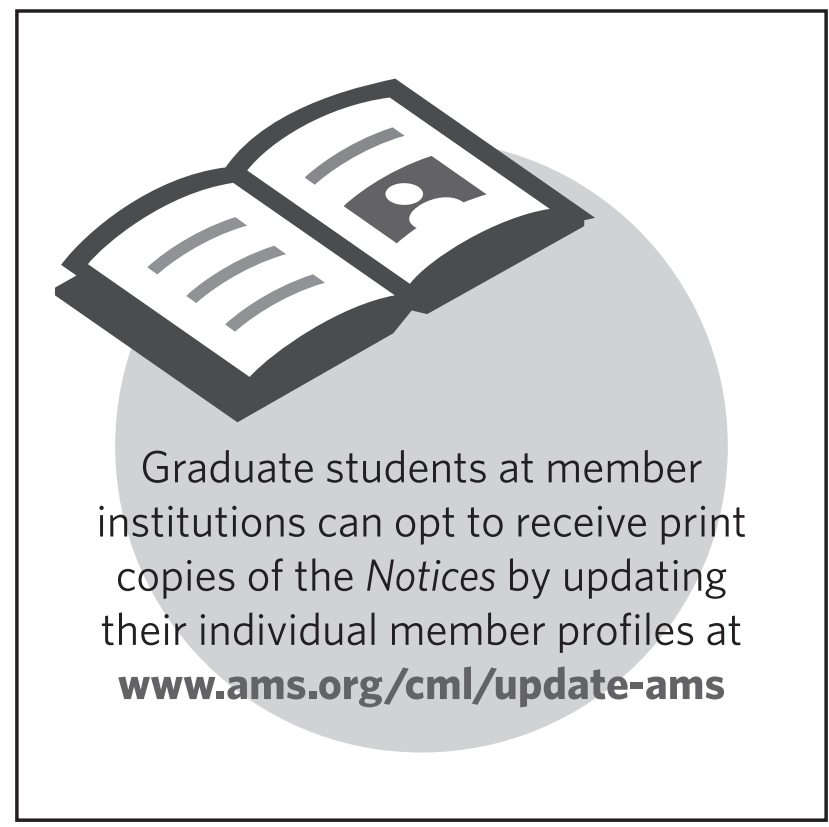

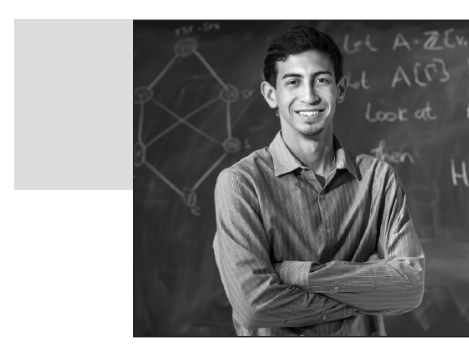

Alexander Diaz-Lopez, having earned his $\mathrm{PhD}$ at the University of Notre Dame, is now visiting assistant professor at Swarthmore College. Diaz-Lopez was the first graduate student member of the Notices Editorial Board. 